\title{
Managing complex long-term conditions and multimorbidity
}

\author{
Author: Archana Dhere ${ }^{\mathrm{A}}$
}

\section{Introduction}

Healthcare systems, medical education and research worldwide are designed to deliver care for individual diseases. However, complex long-term conditions (LTCs) and multimorbidity are on the rise. The existing systems are proving to be inefficient in caring for people with multimorbidity and a wider approach is necessary. ${ }^{1}$ The Royal College of Physicians (RCP) in conjunction with Association of British Clinical Diabetologists (ABCD) organised a conference with the aim to improve understanding of LTCs. Common LTCs like chronic obstructive pulmonary disease (COPD), heart failure, dementia, chronic kidney disease (CKD), diabetes, chronic steroid use, serious mental illness and physical disease, and Parkinson's disease were discussed. The interactive sessions focused on views and experiences of various medical experts in the UK on care pathways, care networks and care models that can help to manage LTCs.

\section{Growing impact and need for change}

LTCs stretch all aspects of health services, as resources struggle to keep up with demand. A 2007 cross-sectional study at 314 medical practices in Scotland revealed $42 \%$ of all primary care patients had one or more morbidities and $23 \%$ were multimorbid. ${ }^{1}$ Hospitals on the edge - a 2012 report by the RCP identified a significant increase in clinical demands on acute services with a $37 \%$ cumulative rise in hospital attendances in the last decade. ${ }^{2}$

About two-thirds of patients admitted to hospital are over 65 years of age. The Future Hospital Commission report, published by the RCP in 2013, highlighted challenges like increasing clinical demands with reduced facilities for acute medicine, changing needs of patients over 65 years of age, poor continuity of care on admission to hospital, inadequate arrangement for out-of-hours care in hospitals and a looming workforce crisis among trainees and consultants that needed to be addressed (Fig 1)..$^{3}$ In 2014, only $60 \%$ of higher specialist trainees were accredited in acute and general internal medicine, and most of these trainees reported unmanageable workload

Author: ${ }^{A}$ ST7 in diabetes and endocrinology, Oxford Centre for Diabetes Endocrinology and Metabolism, Oxford, UK

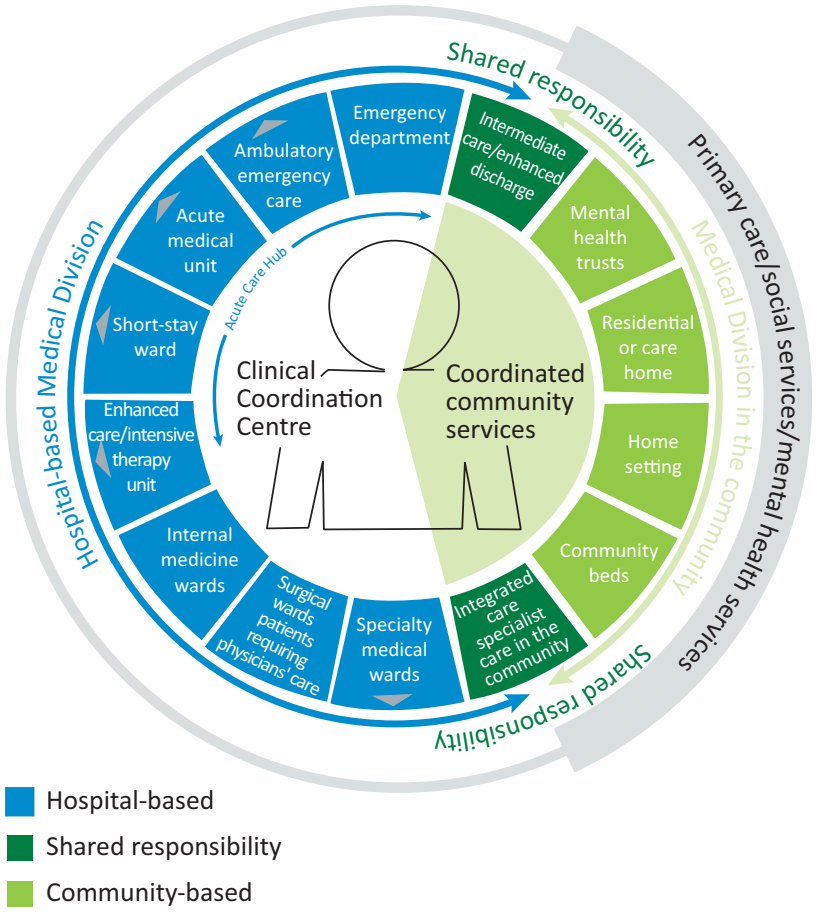

Fig 1. The Medical Division remit: circle of patient-centred care. Directional arrows (in the hospital-based Medical Division) denote areas of the future hospital where patients may be referred on to tertiary specialist care. Reproduced with permission from the Royal College of Physicians. ${ }^{3}$

and poor job satisfaction. Almost $40 \%$ of consultant posts advertised in acute and general internal medicine remained unfilled.

The Shape of training review, presented to the RCP in 2013, identified a need to reshape postgraduate training in the UK. The recommendations included efforts to enhance the value of general internal medicine, increase training numbers in acute and general internal medicine, improve job satisfaction by allowing flexibility in training, and rewards for wider leadership roles. ${ }^{4}$ A new model of care envisioned by the commission aimed at delivering safe, high-quality continuous coordinated care for patients across 7 days and will be possible only if the traditional reactive medical crisis management model is replaced with a holistic psychosocial model that encompasses early proactive support planning to prevent a 


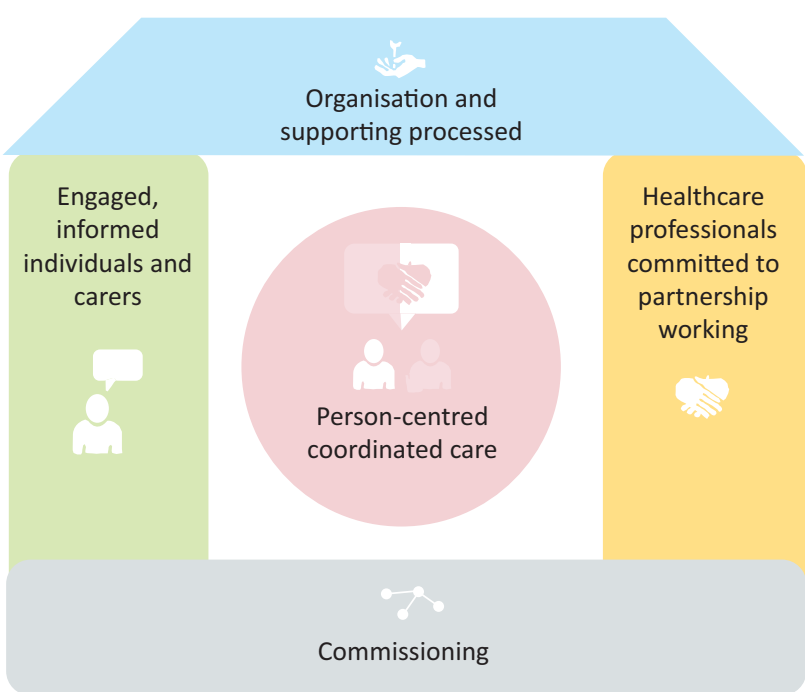

Fig 2. The House of Care. Reproduced from NHS England, www.england.nhs. uk/house-of-care/

crisis. This requires minimising structural divisions between primary, secondary, specialised and social care with improved commissioning, contracting and funding opportunities at various levels of the health and social care system.

The House of Care framework (Fig 2) is one such initiative that makes the patient central to care. The roof of the house is comprised of the best clinical and organisational evidence, the first wall supports professional collaboration between specialists and generalists, the second wall supports patients and their carers to encourage self-care, and the foundations of the house comprises of commissioning groups.

\section{Chronic obstructive pulmonary disease}

Hospitalisation for severe exacerbation of COPD is linked with poor prognosis. The introduction of structured selfmanagement and transitional care plans in the community aims to reduce re-hospitalisation in the first 3 months after discharge. ${ }^{5}$ In addition to specific treatment strategies, like steroids for eosinophilic inflammation, prophylactic macrolides for recurrent exacerbations and volume reduction surgery for emphysema, the national COPD audit and Cochrane review in 2015 showed pulmonary rehabilitation had significant benefits on exercise capacity and quality of life. ${ }^{6}$ Half of COPD patients have one or more comorbidities ${ }^{7}$ and pulmonary rehabilitation was beneficial to those with obesity and congestive heart failure. $^{8,9}$

A Cochrane review in 2014 showed that collaboration between healthcare providers, like nurses, GPs, physiotherapists and chest physicians, to facilitate an integrated disease management programme, including different components like self-management, exercise and nutrition, improves outcomes like dyspnoea, 6-minute walking distance and hospital admissions. ${ }^{9,10}$

\section{Steroids in non-endocrine practice}

Glucocorticoid-induced side effects are reported by $90 \%$ of patients and the impact on patient health and quality of life is dose dependent. ${ }^{11}$ The lowest effective dose of glucocorticoids should be used for the shortest period to avoid the risk of osteoporosis, hyperglycaemia and hypothalamic pituitary axis suppression. Bone density measurement (if glucocorticoids are administered for more than 3 months), early screening and treatment initiation for steroid-induced hyperglycaemia and vigilance for hypothalamic-pituitary axis suppression are recommended.

\section{Dementia and end-of-life care in patients with diabetes}

Diabetes Frail is an emerging concept that explores frailty and its association with diabetes. Frailty is a clinically recognised state of increased vulnerability resulting from ageing and is associated with a decline in the body's physical and psychological reserves. ${ }^{12}$ Frailty increases the level of disability in diabetes. Cognitive impairment and dementia are more common in diabetes and are associated with an increased risk of hypoglycaemia, poor treatment adherence and dependence. ${ }^{13}$ A multidisciplinary assessment of psychosocial and functional capabilities, with medical needs to anticipate risks and devise management strategies, is recommended. The aim is to maintain functional independence, improve the quality of life, and reduce symptoms and medicine burden. ${ }^{14}$ Relaxing glucose control, stopping unnecessary medications, withdrawing statins and pain management at end of life are important.

\section{Chronic kidney disease and diabetes}

Diabetic renal disease is the most common cause of renal failure in the UK. Approximately $20-40 \%$ people with diabetes develop CKD. There is an increased risk of cardiovascular disease in the presence of diabetes and CKD. A multifaceted care program, coordinated through a diabetologist, nephrologist, dietician and primary care physician, is effective in preventing and treating diabetic nephropathy. ${ }^{15}$ The eight pillars of care to focus on are:

1 optimal glycaemic control

2 avoiding extremes of glycaemia

3 monitoring estimated glomerular filtration rate and proteinuria

4 interventions to reduce cardiovascular risk, like smoking cessation, weight reduction, low salt diet, blood pressure control, cholesterol reduction with statin and aspirin prophylaxis

5 foot screening

6 retinopathy screening

7 treatment of anaemia

8 treatment of bone disease. ${ }^{16}$

Management of diabetes in end-stage renal disease remains a challenge because of the rapid changes in glucose homeostasis and altered pharmacokinetics of glucose-lowering medications. Insulin requirement may vary between dialysis and nondialysis days.

\section{Heart failure}

Heart failure prevalence rises as age advances and has a high 5 -year mortality of $45 \% .{ }^{17}$ The all-cause mortality rises in the presence of comorbidities like CKD, anaemia, diabetes and 
COPD. ${ }^{18}$ Treatment strategies for patients should take into account B-type natriuretic peptide (BNP) level, frailty score, nutritional status and whether ejection fraction in heart failure is preserved or reduced.

\section{Mental illness and physical disease}

Physical comorbidities like diabetes mellitus, obesity, COPD and cardiovascular disease are more common in patients with schizophrenia and more likely to be missed. ${ }^{19}$ Schizophrenia is associated with a reduced life expectancy of 11-20 years. ${ }^{20}$ Factors like reduced motivation, side effects from psychotropic medications and an unhealthy lifestyle - including smoking, poor diet, excess alcohol - may contribute to this. Intrauterine environment and childhood adversity play a role. Increased collaboration between mental and physical health services is likely to be helpful.

\section{Parkinson's disease}

Parkinson's disease (PD) is a common neurodegenerative condition affecting 127,000 people in the UK. About $80 \%$ people with PD develop dementia. The National Parkinson's Audit in 2012-2013 identified shortcomings like lack of implementation of best practice, limited access to PD nurses, inadequate patient information in clinics, lack of integration between occupational therapists and speech and language therapists, and lack of training and education. The UK Parkinson's Excellence Network was established in 2015 in an attempt to improve care for people with PD. ${ }^{21}$ The 18 network groups support patients and professionals to build awareness and drive changes in their local area in improving education, research and service development.

\section{Progress so far}

As a part of the NHS Five year forward view, published in 2014, new innovative care models, extra funding arrangements and commissioning approaches have been explored as pilots. The

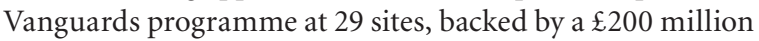
transformation fund for developing new models of care for seamless service across hospitals, primary care, mental health, community nursing, pharmacy and social care, was initiated in March 2015. Since April 2015, integrated personal commissioning has combined the health and social care budgets and is expected to empower patients. In April 2016, Greater Manchester got devolved control of a $£ 6$ billion budget for restructuring and integrating health and social care services based on local needs. The success of these innovations will help determine if these can be used as blueprints for redesigning the entire healthcare system.

\section{References}

1 Barnett K, Mercer SW, Norbury M et al. Epidemiology of multimorbidity and implications for health care, research, and medical education: a cross-sectional study. Lancet 2012;380:37-43.
2 Royal College of Physicians. Hospitals on the edge? The time for action. London: RCP, 2012.

3 Future Hospital Commission. Future hospital: caring for medical patients. London: Royal College of Physicians, 2013.

4 Shape of training. Securing the future of excellent patient care., London: Shape of training, 2013.

5 Suissa S, Dell'Aniello S, Ernst P. Long-term natural history of chronic obstructive pulmonary disease: severe exacerbations and mortality. Thorax 2012;67:957-63.

6 McCarthy B, Casey D, Devane D et al. Pulmonary rehabilitation for chronic obstructive pulmonary disease. Cochrane Database Syst Rev 2015;(2):CD003793.

7 van Manen JG, Bindels PJ, IJzermans CJ et al. Prevalence of comorbidity in patients with a chronic airway obstruction and controls over the age of 40. J Clin Epidemiol 2001;54:287-93.

8 Greening NJ, Evans RA, Williams JE et al. Does body mass index influence the outcomes of a Waking-based pulmonary rehabilitation programme in COPD? Chron Respir Dis 2012;9:99-106.

9 Evans RA, Singh SJ, Collier R et al. Generic, symptom based, exercise rehabilitation; integrating patients with COPD and heart failure. Respir Med 2010;104:1473-81.

10 Kruis AL, Smidt N, Assendelft WJ et al. Cochrane corner: is integrated disease management for patients with COPD effective? Thorax 2014;69:1053-5.

11 Curtis JR, Westfall AO, Allison J et al Population-based assessment of adverse events associated with long-term glucocorticoid use. Arthritis Rheum 2006;55:420-6.

12 British Geriatrics Society. Fit for frailty: consensus best practice guidance for the care of older people living with frailty in community and outpatient settings. London: British Geriatrics Society, 2014.

13 Sinclair AJ, Hillson R, Bayer AJ, National Expert Working Group. Diabetes and dementia in older people: a Best Clinical Practice Statement by a multidisciplinary National Expert Working Group. Diabet Med 2014;31:1024-31.

14 Sinclair A, Dunning T, Rodriguez-Mañas L. Diabetes in older people: new insights and remaining challenges. Lancet Diabetes Endocrinol 2015;3:275-5.

15 Hahr AJ, Molitch ME. Management of diabetes mellitus in patients with chronic kidney disease. Clin Diabetes Endocrinol 2015;1:2.

16 Kidney Disease Improving Global Outcomes (KDIGO). KIDIGO 2012 clinical practice guideline for the evaluation and management of chronic kidney disease. Kidney International Supplements 2013;3:1-163.

17 Mitchell P, Marle D, Donkor A et al. National heart failure audit April 2013-March 2014. London: UCL, 2014.

18 van Deursen VM, Urso R, Laroche C et al. Co-morbidities in patients with heart failure: an analysis of the European Heart Failure Pilot Survey. Eur J Heart Fail 2013;16:103-11.

19 Smith DJ, Langan J, McLean G, Guthrie B, Mercer SW. Schizophrenia is associated with excess multiple physical-health comorbidities but low levels of recorded cardiovascular disease in primary care: cross-sectional study. BMJ Open 2013;3:e002808.

20 Laursen TM, Nordentoft M, Mortensen PB. Excess Early Mortality in Schizophrenia. Annu Rev Clin Psychol 2014;10:425-48.

21 Burn D. UK Parkinson's Excellence Network: empowering service improvement across the UK. Neurodegener Dis Manag 2015;5:173-6.

Address for correspondence: Dr A Dhere, Oxford Centre for Diabetes, Endocrinology \& Metabolism, Oxford University Hospitals NHS Trust, Churchill Hospital, Headington, Oxford OX3 7LE, UK.

Email: archanadhere@doctors.org.uk 